\title{
Nanofibrous Scaffold for Bone Tissue Engineering via AC Electrospinning
}

\author{
Radek Jirkovec, Tomas Kalous, Pavel Holec, Alzbeta Samkova, Jiri Chvojka \\ Technical University of Liberec \\ Studentska 1402/2, Liberec, Czech Republic \\ radek.jirkovec@tul.cz
}

\section{Extended Abstract}

The aim of this work was to develop and test micro / nano fiber material (scaffold) designed for tissue engineering, especially for bone regeneration. In the work the aim was to find a suitable polymeric material to create a suitable structure using alternating current (AC) spinning technology and to test mechanical properties and biocompatibility of scaffolds.

For bone regeneration are used scaffolds that are physically chemically, structurally and biologically similar to extracellular matrix. The scaffold properties, such as porosity, affect cell proliferation, differentiation, and bone regeneration itself. For bone regeneration are used both natural and synthetic polymers, such as collagen, gelatin, chitosan, polylactic acid, polycaprolactone, and others. [1, 2, 3, 4] The polycaprolactone, which promotes cellular viability, has been selected from the previous experiments, where we used fibrous layer formed by direct current (DC) spinning.

As it mentioned in the introduction, the production of micro / nano fiber scaffolds was carried out using AC spinning technology. It is a high-voltage technology which used, in comparison to DC spinning, a frequency of tens of Hz. The formed fibers are provided with both positive and negative charges. Their interaction creates a neutral fibrous bundle that can form in free space. The alternating voltage allows the formation of nanofibres without the anti-electrode, whereby the nanofibers formed can be deposited on the core core yarn, the rotary cylinder or the static collector. Compared to DC spinning, this method produces a bulky, fluffy layer. [5, 6, 7]

The first phase of the experiment was to find a suitable solvent system and concentration of the polymer solution. The experiment was performed with two molecular weights of polycaprolactone: Mn 45,000 and Mn 80,000. Three solvent systems were chosen for the experiment: chloroform:ethanol; chloroform:ethanol:acetic acid; and acetic acid:formic acid:acetone. The experiment led to finding that the solution prepared in the chloroform: ethanol solvent system was unsuitable, spinning was poor. In the second solvent system, the spinning process was good but with low production. The spinning with the last solvent system was optimal and with high production. Therefore, the solvent system acetic acid: formic acid: acetone was chosen for further testing.

The second phase of the experiment was aimed at producing of fiber layers with different surface density. The spinning was carried out on a rotating drum, the surface density was controlled by the spinning time. However, an important parameter is the rotation speed of the rotating drum, which affects the stiffness of the resulting layer. Thus, two rotation speeds of the rotating drum were used during the experiment to compare the strengths of the resulting layers.

In the last stage of the experiment, for biological testing were spun two fiber layers with selected spinning parameters, where one fiber layer was functionalized with hydroxyapatite to increase cell viability. Biological testing was performed with human MG-63 osteoblasts.

In the experiment was found a suitable solvent system, and it was optimized the spinning process. Layers with different surface density and different rigidity were produced and subjected to mechanical testing. The highest strength layer and the hydroxyapatite functionalized layer were selected for biological testing. During biological experiment, the layers have been shown not to be cytotoxic and promote cell viability, the layer with the hydroxyapatite has shown a higher rate of cell proliferation using CCK-8 method.

\section{References}

[1] Chen Shi, Zhangqin Yuan, Fengxuan Han, Caihong Zhu, Bin Li, "Polymeric biomaterials for bone regeneration," Annals of Joint, vol. 1, no. 9, 2016.

[2] Fergal J. O'Brien, "Biomaterials \& scaffolds for tissue engineering,“"Materials Today, vol. 14, no. 3, pp. 88-95, 2011. 
[3] T. Ghassemi, A. Shahroodi, M. H. Ebrahimzadeh, A. Mousavian, J. Movaffagh, A. Moradi, "Current Concepts in Scaffolding for Bone Tissue Engineering," The archives of bone and joint surgery, vol. 6, no. 2, pp. 90-99, 2018.

[4] Jakub Erben, Vera Jencova, Jiri Chvojka, Lenka Blazkova, Katerina Strnadova, Miroslav Modrak, Eva Kuzelova Kostakova, "The combination of meltblown technology and electrospinning - The influence of the ratio of micro and nanofibers on cell viability," Materials Letters, vol. 173, pp. 153-157, 2016.

[5] Attila Balogh, Richárd Cselkó, Balázs Démuth, Geert Verreck, Jürgen Mensch, György Marosi, Zsombor Kristóf Nagy, "Alternating current electrospinning for preparation of fibrous drug delivery systems, " International Journal of Pharmaceutics, vol. 495, no. 1, pp. 75-80, 2015.

[6] Jan Valtera, Tomas Kalous, Pavel Pokorny, Ondrej Batka, Martin Bilek, Jiri Chvojka, Petr Mikes, Eva Kuzelova Kostakova, Petr Zabka, Jana Ornstova, Jaroslav Beran, Andrei Stanishevsky, David Lukas, "Fabrication of dualfunctional composite yarns with a nanofibrous envelope using high throughput AC needleless and collectorless electrospinning," Scientific Reports, vol. 9, no. 1, 2019

[7] Andrei Stanishevsky, W. Anthony Brayer, Pavel Pokorny, Tomáš Kalous, David Lukáš, "Nanofibrous alumina structures fabricated using high-yield alternating current electrospinning," Ceramics International, vol. 42, no. 15, pp. 17154-17161, 2016. 